\title{
Do Turkish Gynecologists and Obstetricians recommend pregnant women the influenza vaccine?
}

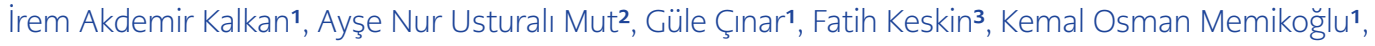 \\ Alpay Azap ${ }^{1}$ \\ 1 Department of Infectious Diseases and Clinical Microbiology, Ankara University School of Medicine, Ankara, Turkey \\ 2 Department of Public Health, Ankara University School of Medicine, Ankara, Turkey \\ 3 Department of Obstetrics and Gynecology, Edirne Sultan 1. Murat Hospital, Edirne, Turkey
}

\begin{abstract}
Objective: Influenza is a systemic infectious disease. It is recommended that all pregnant women receive the influenza vaccine because the mortality of the disease is high during pregnancy. However, the rates of influenza vaccination in pregnant women are low. This study aims to estimate the rate at which Turkish gynecologists and obstetricians (GOs) recommend the influenza vaccine to their pregnant patients.. Materials and Methods: This study was designed as a cross-sectional survey. The sample size was calculated to be 364 based on 95\% confidence interval and 5\% margin of error. The data were collected through a questionnaire consisting of 17 questions, which was distributed through social media. The final study group included in the research consisted of 384 GOs.

Results: The mean age of the GOs that participated in the study was 39.7 years $(S D=10.2)$. $43.5 \%$ of the GOs reported that they recommended the influenza vaccine to pregnant women who had consulted with them, while $62.8 \%$ reported that at least $50 \%$ of the pregnant women they had recommended the vaccine rejected it. According to multivariate logistic regression analysis, three factors increased the rate of GOs' not to recommend influenza vaccination: the GOs' age, not receiving influenza vaccination themselves, and not knowing the social insurance reimbursement for the vaccine.

Conclusion: Vaccination of pregnant women is necessary because of the increased mortality risk of influenza during pregnancy. Even though GOs are not in charge of vaccinating their patients during their routine pregnancy follow-up, they can contribute to vaccination rates by recommending the vaccine. Physicians' application of scientific knowledge and transferring it to their patients can contribute to increased adult immunization rates.

Keywords: Influenza, Influenza Vaccine, Pregnancy, Vaccination, Viral Infections
\end{abstract}

\section{INTRODUCTION}

I nfluenza is a systemic infectious disease. Many groups, including patients with concomitant chronic heart and lung diseases, immunosuppressed individuals, people over 50 years old, and all health staff are considered at-risk groups for influenza (1). Pregnant women are particularly considered to be at a higher risk. Influenza infection
Corresponding Author: Irem Akdemir Kalkan

E-mail:

iremakd@yahoo.com

Received: April 14, 2020 Accepted: April 30, 2020 Published: April 30, 2020

\section{Suggested citation:}

Kalkan IA, Mut ANU, Çınar G, Keskin F, Memikoğlu KO, Azap A. Do Turkish Gynecologists and Obstetricians recommend pregnant women the influenza vaccine? Infect Dis Clin Microbiol 2020; 1: 16-23.

DOI: $10.36519 / \mathrm{idcm} .2020 .0005$ 
during pregnancy is associated with increased morbidity and mortality and fetal growth retardation. Administering influenza vaccines during pregnancy protects the pregnant woman, the fetus, and the newborn (until the sixth month). The influenza vaccine in its inactive form can be administered safely throughout the pregnancy period, and it protects against three to four influenza viruses depending on the content of the vaccine. The World Health Organization, the Centers for Disease Control and Prevention and the health authorities of several countries recommend vaccination for all women who plan to get pregnant or who are pregnant during the influenza seasons. However, influenza vaccination rates are low all over the world among both in pregnant women and all other risk groups (1-3).

In Turkey, adult immunization services are recommended within the framework of the Extended Immunization Program (4). The influenza vaccination rates among health staff, people with chronic lung disease, and the diabetic population in Turkey are low, while pregnant women's influenza vaccination rate is unknown. The influenza vaccination rates can, however, be estimated through the number of vaccines done in a season at the primary health-care centers. Pregnant women see gynecologists and obstetricians (GOs) at regular intervals, which provides an opportunity to recommend and administer vaccines during pregnancy. Recognizing how this opportunity is used in practice and identifying the reasons why physicians do not recommend the vaccine could help to design strategies to increase immunization rates (5-6). To the best of our knowledge, no study has investigated this topic in Turkey. In this study, we inquired into the rates of Turkish GOs' recommendation of influenza vaccines to pregnant women and the factors that discourage the GOs from recommending the vaccine.

\section{MATERIALS AND METHODS}

This study was a cross-sectional survey. The study group consisted of GOs, including both residents and specialists who were working in Turkey at the time the study was conducted. Based on the records of the Ministry of Health, the total number of active GO residents and consultants in Turkey as of December 2018 was 7,021. When the rate of influ- enza vaccine recommendation to pregnant women among the GOs (i.e., the dependent variable) was set at $50 \%$, a confidence interval of $95 \%$, and a margin of error of $5 \%$, a minimum sample size of 364 was calculated using the Epi Info program. Thus, a total of 384 GOs were included in the study group. The stratified sampling method was used to choose a representative sample of the GOs by geographical area. To distribute the sample group by population and geographic region, survey responses that disrupted the stratification were excluded from the dataset by taking into account the date and time of the survey.

As it was not possible to reach all the GOs in Turkey, the sample population was not selected from the list. Instead, the data collection phase was carried out through a closed social media group with a membership of 4,300 GOs. The data were collected between March 2019 and May 2019 via an online questionnaire, which comprised 17 questions. The questionnaire was created using Google Forms. The questions were arranged according to the question content and the answer type; both multiple-choice questions and open-ended questions were asked (see Table 1). GOs who had teaching duties and scientific afiliations were considered as academicians.

\section{Statistical Analysis}

The data were analyzed using IBM SPSS Statistics

\section{HIGHLIGHTS}

- About $44 \%$ of the GOs recommend influenza vaccines to pregnant women who consult with them.

- According to the data we collected, approximately $80 \%$ of the GOs did not receive the influenza vaccine in 2019.

- Three factors increased the rate of GOs' not to recommend influenza vaccination: the GOs' age, not receiving influenza vaccination themselves, and not knowing the social insurance reimbursement for the vaccine.

- About $63 \%$ of the GOs who recommended the influenza vaccine reported that at least $50 \%$ of the pregnant women refused the vaccine. 
Table 1. Questions directed at obstetricians and gynecologists.

\begin{tabular}{|c|c|}
\hline 1. & What is your age? \\
\hline 2. & What is your gender? \\
\hline 3. & Which region is your institution located in? \\
\hline 4. & What is your title? \\
\hline 5. & How long have you been an obstetrician and a gynecologist? \\
\hline 6. & Which type of institution do you work for? \\
\hline 7. & Do you plan to or are you planning to have an influenza vaccine this year? \\
\hline 8. & Do you know the mortality rate of influenza during pregnancy? \\
\hline 9. & What is the mortality rate of influenza during pregnancy? \\
\hline 10. & Do you recommend the influenza vaccine to pregnant women who apply to you? \\
\hline 11. & How do you recommend the influenza vaccine to pregnant women? \\
\hline 12. & In which trimester do you recommend the influenza vaccine to pregnant women who apply to you? \\
\hline 13. & What percentage of pregnant women to whom you recommended the influenza vaccine rejected it? \\
\hline 14. & What are the most common reasons pregnant women give for refusing the influenza vaccine? \\
\hline 15. & What do you do if you encounter a pregnant woman who refuses to receive the influenza vaccine? \\
\hline 16. & What are the reasons for not recommending the influenza vaccine to pregnant women? \\
\hline 17. & Is the influenza vaccine for pregnant women covered by reimbursement in this country? \\
\hline
\end{tabular}

21.0. The descriptive findings and percentage distributions were given with the mean, standard deviation (SD), and minimum and maximum values. Chi-square test and backward stepwise multivariate logistic regression analysis were used to analyze the relationship between not recommending the influenza vaccine and the independent variables. Statistical significance was set as $\mathrm{p}<0.05$.

\section{RESULTS}

The mean age of the of the study group was 39.7 years $(\mathrm{SD}=10.2$; minimum $=24$ years; maxi- mum $=73$ years). Fifty-seven per cent of the GOs were females. Regarding the distribution by region, $36.5 \%$ of the GOs were from the Marmara region, while 18.2\% were from Central Anatolia (Table 2). The mean duration of work among the residents was 2.37 years $(\mathrm{SD}=1.46$; minimum $=0.01$ years; maximum $=5$ years), but it was 12.12 years $(S D=$ 10.11; minimum $=0.08$ years; maximum $=51$ years) among the consultants.

About $80.7 \%$ of the GOs in the study group reported that they had not received or did not intend to receive the influenza vaccine in 2019. Only about $41.7 \%$ 
Table 2. Status of the respondents regarding the recommendation of influenza vaccine according to gender, geographical region, title, and institution

\begin{tabular}{|c|c|c|c|c|c|c|c|}
\hline \multirow{3}{*}{ Variable } & \multicolumn{4}{|c|}{ Recommendation of influenza vaccine } & \multirow{2}{*}{\multicolumn{2}{|c|}{ Total }} & \multirow{3}{*}{ p-value } \\
\hline & \multicolumn{2}{|c|}{ Recommend } & \multicolumn{2}{|c|}{ Do not recommend } & & & \\
\hline & $\mathbf{N}$ & $\%$ & $\mathbf{N}$ & $\%$ & $\mathbf{N}$ & $\%$ & \\
\hline \multicolumn{8}{|l|}{ Gender } \\
\hline Female & 90 & 41.1 & 129 & 58.9 & 219 & 57.0 & \multirow{2}{*}{0.299} \\
\hline Male & 77 & 46.7 & 88 & 53.3 & 165 & 43.0 & \\
\hline \multicolumn{8}{|l|}{ Geographic region } \\
\hline Marmara Region & 69 & 49.3 & 71 & 50.7 & 140 & 36.5 & \multirow{7}{*}{0.011} \\
\hline Central Anatolia Region & 39 & 55.7 & 31 & 44.3 & 70 & 18.2 & \\
\hline Aegean Region & 21 & 39.6 & 32 & 60.4 & 53 & 13.8 & \\
\hline Mediterranean Region & 19 & 40.4 & 28 & 59.6 & 47 & 12.2 & \\
\hline South East Anatolia Region & 8 & 26.7 & 22 & 73.3 & 30 & 7.8 & \\
\hline Black Sea Region & 7 & 28.0 & 18 & 72.0 & 25 & 6.5 & \\
\hline East Anatolia Region & 4 & 21.1 & 15 & 78.9 & 19 & 4.9 & \\
\hline \multicolumn{8}{|l|}{ Title } \\
\hline Resident & 34 & 44.7 & 42 & 55.3 & 76 & 19.8 & \multirow{3}{*}{0.001} \\
\hline Consultant & 111 & 39.8 & 168 & 60.2 & 279 & 72.7 & \\
\hline Academician & 22 & 75.9 & 7 & 24.1 & 29 & 7.6 & \\
\hline \multicolumn{8}{|l|}{ Institution } \\
\hline Government hospital & 39 & 35.8 & 70 & 64.2 & 109 & 28.4 & \multirow{4}{*}{0.077} \\
\hline University & 30 & 49.2 & 31 & 50.8 & 61 & 15.9 & \\
\hline Research hospital & 45 & 52.9 & 40 & 47.1 & 85 & 22.1 & \\
\hline Private hospital & 53 & 41.1 & 76 & 58.9 & 129 & 33.6 & \\
\hline Total & 167 & 43.5 & 217 & 56.5 & 384 & 100 & \\
\hline
\end{tabular}


If pregnancy coincided with the influenza season (October-May)

According to the underlying disease of the pregnant woman

If influenza vaccine comes to GO's mind

For every pregnant woman

According to the trimester status

Based on the pregnant woman's attitude toward the vaccine

According to each pregnant woman's sociocultural and economic status

If the pregnant is at an advanced maternal age

If the pregnancy was due to in vitro fertilization

$n=167$

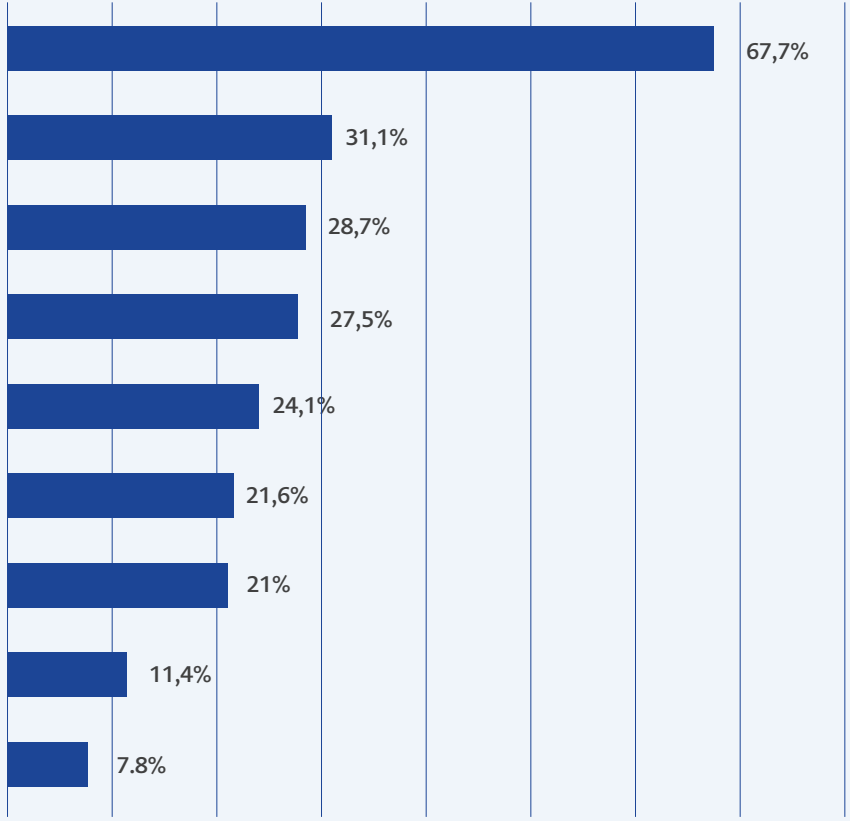

Figure 1. The situations when the gynecologists and obstetricians recommend the influenza vaccine

Because the vaccines were followed up in family health centers

Because of problems during pregnancy that may be a result of the vaccination

Due to intensive working conditions

Influenza vaccine is not effective

Because influenza vaccination for pregnant women is not administered at my institution

Because of the cost of influenza vaccine

Because influenza vaccination harms pregnant women or fetus

Because I do not trust vaccines

Influenza vaccine is contraindicated during pregnancy

$$
n=217
$$

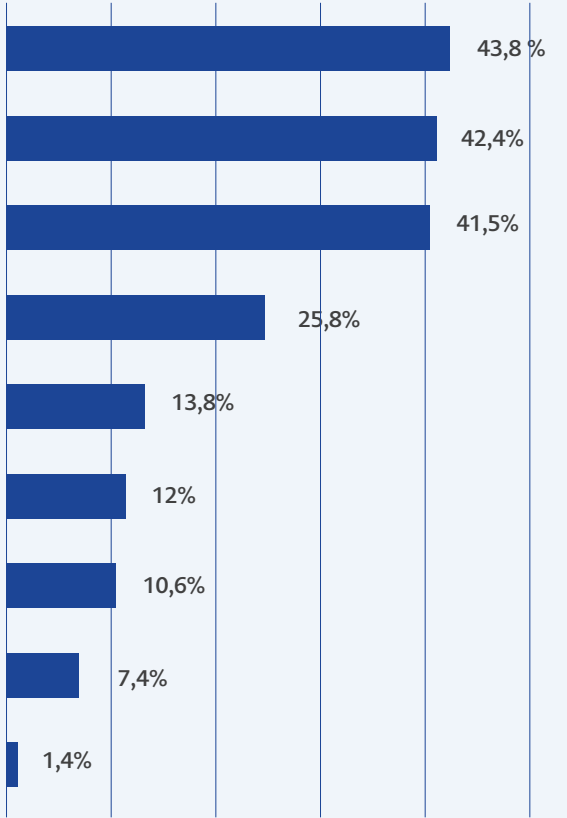

0

Figure 2. The reasons for not recommending influenza vaccination for pregnant women 
Table 3. Status of the respondents regarding the recommendation of influenza vaccine to pregnant women according to the influenza vaccine status of the respondent, influenza mortality rate and influenza vaccine reimbursement

\begin{tabular}{|c|c|c|c|c|c|c|c|}
\hline \multirow{3}{*}{ Variable } & \multicolumn{4}{|c|}{ Recommendation of influenza vaccine } & \multirow{2}{*}{\multicolumn{2}{|c|}{ Total }} & \multirow{3}{*}{ p-value } \\
\hline & \multicolumn{2}{|c|}{ Recommend } & \multicolumn{2}{|c|}{ Do not recommend } & & & \\
\hline & $\mathbf{N}$ & $\%$ & $\mathbf{N}$ & $\%$ & $\mathbf{N}$ & $\%$ & \\
\hline \multicolumn{8}{|c|}{ Received or has been thinking about receiving the influenza vaccine } \\
\hline Yes & 58 & 78.4 & 16 & 21.6 & 74 & 19.3 & \multirow{2}{*}{$<0.001$} \\
\hline No & 109 & 35.2 & 201 & 64.8 & 310 & 80.7 & \\
\hline
\end{tabular}

Knowledge of the mortality rate of influenza during pregnancy

\begin{tabular}{lcccccc}
\hline Do not know & 81 & 36.2 & 143 & 63.8 & 224 & 58.3 \\
\hline Under 10\% & 44 & 46.3 & 51 & 53.7 & 95 & 24.7 \\
\hline Over $\mathbf{1 0 \%}$ & 42 & 64.6 & 23 & 35.4 & 65 & 16.9 \\
\hline
\end{tabular}

Knowledge of whether influenza vaccine is covered by reimbursement for pregnant women

\begin{tabular}{lcccccc}
\hline Yes & 93 & 76.2 & 29 & 23.8 & 122 & 31.8 \\
\hline No & 30 & 30.0 & 70 & 70.0 & 100 & 26.0 \\
\hline Do not know & 44 & 27.2 & 118 & 72.8 & 162 & 32.2 \\
\hline Total & 167 & 43.5 & 217 & 56.5 & 384 \\
\hline
\end{tabular}

of them stated that they knew the mortality rate of influenza during pregnancy (Table 2). The mean mortality rate of influenza during pregnancy according to the GOs was $14.6 \%$ (SD = 15.1; minimum = $0.1 \%$; maximum $=90 \%$ ).

About $43.5 \%$ of the GOs reported that they recommended the influenza vaccine to pregnant women who consulted with them, and $67.7 \%$ of them stated that they recommended the vaccine only when the pregnancy coincides with the influenza season (October-May) (Figure 1). Regarding the schedule for vaccination, $17.2 \%$ of the GOs reported they recommended the influenza vaccine in both the second and third trimesters of pregnancy, while $14.8 \%$ stated they recommended it at any time during pregnancy. About $11.4 \%$ of the GOs who recommended the influenza vaccine had not encountered a pregnant woman who rejected the vaccine, while $62.8 \%$ reported that at least $50 \%$ of the pregnant women refused the vaccine when they recommended it. When asked the most common reasons for the rejection of influenza vaccination, $74.3 \%$ of the GOs reported that the pregnant women thought that the vaccine would harm them and their babies, while $20.4 \%$ of the GOs reported that the pregnant women who rejected the influenza vaccine were vaccine-hesitant people. Furthermore, $15.6 \%$ of the GOs reported that their pregnant patients did not receive the vaccine because of their spouses, while $12.6 \%$ stated that their patients refused the vaccine because their previous doctors did not recommend it. About $68.9 \%$ of the GOs who recommended the influenza vaccine stated that they tried to convince their patients by explaining the medical necessity of the vaccine. About $29.3 \%$ of the GOs stated that they prefer not to recommend the vaccine.

Approximately $56.5 \%$ of the GOs stated that they do not recommend the influenza vaccine to pregnant 
women who consult with them. $43.8 \%$ of the GOs stated that since the vaccines are usually administered and followed up at the family health centers they do not think about vaccines (Figure 2). Among the GOs, only about $31.8 \%$ were aware that social insurance reimbursement covers the influenza vaccine for pregnant women in Turkey.

The distribution of the respondents (i.e., the GOs) regarding the recommendation of the influenza vaccine according to independent variables, is given in Tables 2 and 3. According to the multivariate logistic regression analysis, the probability that a GO would not recommend the influenza vaccine increased 1.043 (1.008-1.080) times with each unit increase in the GO's age. The probability that a GO would not recommend the influenza vaccine was higher in GOs working in the Mediterranean $(\mathrm{OR}=2.549 ; 1.048-6.203)$, Southeast $(\mathrm{OR}=4.562$; 1.486-11.008), and Eastern Anatolia ( $\mathrm{OR}=5.942$; 1.484-13.781) regions than in GOs working in Marmara region. The GOs who did not receive influenza shot themselves (OR $=7.897 ; 3.895-16.013)$, the GOs who did not know that influenza vaccine was covered for reimbursement in Turkey $(\mathrm{OR}=12.330$; 6.281-24.204), and the GOs who thought the vaccine was covered for reimbursement $(\mathrm{OR}=7.526$; 3.767-15.035) had lower rates of recommending the vaccine than other GOs. Whereas, the rate of recommending the vaccine was higher among GOs who stated that they knew the rate of influenza mortality in pregnancy $(\mathrm{OR}=0.415 ; 0.189-0.911)$.

\section{DISCUSSION}

Awareness of the importance of adult immunization has increased in recent years and has shaped health policies and approaches. However, the role of preventive medicine physicians in recommending the vaccines has been hampered by intensive working conditions and the shifting demands of therapeutic practice, which has led to a lack of adult immunization services.

Although previous research has suggested that influenza vaccines are recommended to high-risk populations at a higher rate, the vaccination rates are still quite low (5-7). In all the studies evaluated, the rate of influenza vaccination in all at-risk groups was below 50\% (8-10). The prevalence of influenza vaccine recommendation rates among the GOs was found to be $43.5 \%$ in this study, which makes the well-being of the fetus a serious concern because of the increased risk of mortality. Factors that made the GOs less likely to recommend the vaccine include their assessment of the risk of influenza during pregnancy and the comorbid diseases of the pregnant woman. The percentage of the GOs who recommended the vaccine in all cases as recommended by the Centers for Disease Control and Prevention was only $27.5 \%$.

More than half of the GOs stated that at least 50\% of the pregnant women they recommended the vaccine to refused it mainly because they had the fear that the vaccine could be harmful to their pregnancy. Vaccine hesitation and rejection are critical issues that threaten serious social consequences all over the world, including in Turkey. This study revealed that, even though some of the GOs recommended the vaccination, a considerable proportion of the pregnant women were reluctant to receive it. About $50 \%$ of the GOs reported that pregnant women were afraid to receive the vaccination due to negative comments from the media that suggest that the vaccine might cause harm. This indicates that pregnant women are exposed to serious misinformation on the important subject of vaccination.

In a survey conducted among 1,061 health professionals (physicians, nurses, and midwives) caring for pregnant women in Canada, both the vaccination rate $(85 \%)$ and the rate by which physicians recommend the vaccine to pregnant women $(69 \%)$ were found to be high. In the same study, the health professionals reported that $30 \%$ of the pregnant women they recommended vaccines to refused it. Although this percentage is lower than in our study, the reason why pregnant women refused the vaccination is similar: the fear that the vaccine can harm the fetus (11).

In our study, factors such as increased physician age, not knowing that the vaccine is covered by reimbursement, and not knowing the mortality rate of the disease were found to be associated with lower vaccine recommendation rates. These findings reveal that vaccination recommendation rates 
are lower when the level of knowledge and awareness about the disease is low. In almost all studies examining the problems of adult immunization, intensive working conditions of physicians and lack of easy access to vaccines have been identified as important obstacles to higher vaccination rates $(12,13)$. In this study, we confirmed that some physicians did not recommend vaccination due to the intense nature of their work and because they did not know that the vaccine was covered for reimbursement, which contributed to the low vaccination rates.
The subject of immunization should be an important part of both medical education and speciality training. It is a dynamic subject that requires innovation, especially for the vaccination of special patient groups. The introduction of new vaccines and vaccination schemes requires that all physicians closely monitor this field and renew their knowledge at the postgraduate level (14). Influenza vaccination is very important during pregnancy, as it contributes to a healthy pregnancy process, healthy infant follow-up, and overall public health. Only by educating physicians will it be possible to achieve high adult influenza immunization rates.
Informed Consent: Written consent was obtained from the patient.

Ethical Approval: The Ethical Committee of Ankara University Non-Clinical Research Ethics Committee on Humans approved the study with the decision number of 21/03/2019-137.

Peer-review: Externally peer-reviewed

Author Contributions: Concept - İ.A.K.; Design - İ.A.K., A.U.U.M., G.Ç., F.K.; Supervision - İ.A.K., A.U.U.M.,F.K.; Data Collection and/or
Processing - A.U.U.M.,F.K. ; Analysis and/or Interpretation - İ.A.K., A.U.U.M., A.A., K.O.M.; Literature Review - İ.A.K., G.Ç.; Writer I.A.K.,G.Ç., K.O.M., A.A. ; Critical Reviews - G.C.., F.K., K.O.M., A.A.

Conflict of Interest: The authors have no conflict of interest to declare.

Financial Disclosure: The authors declared that this study has received no financial support.

\section{REFERENCES}

1 Paules C, Subbarao K. Influenza. Lancet 2017; 390: 697-708.

2 Grohskopf LA, Sokolow LZ, Broder KR, Olsen SJ, Karron RA, Jernigan DB, et al. Prevention and Control of Seasonal Influenza with Vaccines. MMWR Recomm Rep 2016; 65: 1-54.

3 Rasmussen SA, Jamieson DJ, Uyeki TM. Effects of influenza on pregnant women and infants. Am J Obstet Gynecol. 2012; 207: S3-8.

4 Turkish Ministry of Health. Circular about Extended National Immunization Program, 25.02.2008;6111;2008/14.

5 Gürbüz Y, Tütüncü EE, Şencan İ, Şendağ E, Callak F, Sevinç G, et al. Study on the willingness of health care workers to receive an influenza vaccination during the 2009 Influenza A (H1N1) pandemic. Pam Med J 2013; 6:12-17.

6 Biberoğlu K, Biberoğlu S, Özbakkaloğlu M, Bilgir O, Aslan L, Bolaman Z, et al. Come on Adults, Let's Vaccinate! The Report of Turkish Internal Specialist Association- Aegean Working Group. Available at: URL: http://www.tihud.org.tr/main/content? ref=2\&child=179. Accessed 30 June, 2019.

7 Stark LM, Power ML, Turrentine M, Samelson R, Siddigui MM, Paglia MJ, et al. Influenza vaccination among pregnant women: patient beliefs and medical provider practices. Infect Dis Obstet Gynecol 2016; 2016: 3281975.

8 Callaghan WM, Creanga AA, Jamieson DJ. Pregnancy-Related
Mortality Resulting From Influenza in the United States During the 2009-2010 Pandemic. Obstet Gynecol. 2015;126(3):486-490.

9 Ding H, Black CL, Ball S, Fink RV, Williams WW, Fiebelkorn AP, et al. Influenza vaccination coverage among pregnant women - United States, 2016-17 influenza season. MMWR Morb Mortal Wkly Rep 2017; 66: 1016-1022.

10 Ulusoy E, Arkan D. Rates of influenza vaccination of healthcare workers working in pediatric units. Turkiye Klinikleri J Nurs. 2010; 2: 11-15.

11 Dube E, Gagnon D, Kaminsky K, Green CR, Quakki M, Bettinger JA, et al. Vaccination Against influenza in pregnancy: a survey of Canadian Maternity Care Providers. J Obstet Gynaecol Can 2019; 4: 479-488.

12 Alici DE, Sayiner A, Unal S. Barriers to adult immunization and solutions: Personalized approaches. Hum Vaccin Immunother 2017; 13: 213-215

13 Toprak D, Köksal İ, Sargın M, Akan H. Adult vaccination, problems in practice and solution proposals, role of family physicians in adult vaccination. Türk Aile Hek Derg 2018; 22:166-174.

14 Akdemir-Kalkan İ, Çelen MK. Assessment of hepatitis B vaccination and HBV infection in family practitioners in Batman province. Flora. 2018; 23: 227-231. 